\title{
- Digital architectural visualization
}

\section{Alexander Koutamanis}

Faculty of Architecture, Delft University of Technology

\section{- Abstract}

\section{Visualization and architecture}

- Roles of visualization

The democratization of computer technologies

Visualization and modelling

- Representation

Implementation mechanisms

Design modelling

Abstraction and specificity

Compound representations 
Realism: visual inspection and
analysis

- Projecting appearances Beyond intuition: scientific visualization

Dynamic visualization

Future developments

References 


\section{Abstract}

The traditional emphasis of architectural education and practice on spatial visualization has contributed to the development of an overtly visual architectural culture which agrees with our predominantly visual interaction with the built environment. The democratization of computer technologies is changing architectural visualization in two significant ways. The first is that the availability of affordable, powerful digital versions of analogue visual media and of new, complementary techniques is facilitating the application of computer visualization in most aspects of the design and management of the built environment. The second is the opening of a wide and exciting new market for visualization in information systems, for example through interfaces that employ spatial metaphors, which arguably are extensions of the three dimensional structures the architect knows better than other design specialists of today.

The transition from analogue to digital visualization poses questions that encompass the traditional investigation of relationships between geometric representations and built form, as well as issues such as a unified theory of architectural representation, the relationships between analysis and visualization and the role of abstraction in the structure of a representation. In addition to theoretical investigations, the utilization of new possibilities in architectural visualization requires technology and knowledge transfer from areas other than computer science. The integration of such transfers suggests flexible, modular approach which contradicts the holistic, integral principles of computer-aided architectural design. 


\section{Visualization and architecture}

\section{Roles of visualization}

Visualization of real and imaginary space has been a traditional strong point of architectural education and practice. Even when architectural design is removed from the influence of the visual arts, the architect makes extensive and intensive use of visual methods and techniques in the development of a composition, the specification of a design product, the communication of more abstract concepts and the analysis of design ideas. As a result, our knowledge of world architecture stems more from published photographs and drawings than from personal experience (Evans 1989).

The emphasis on matters visual in architecture is not accidental. Human interaction with the natural and built environment is predominantly visual. A wide spectrum of human activities, from aesthetic appreciation to the planning of actions relies heavily on visual information and makes use of visual means to analyse and formulate states and conclusions. Visualization has been a significant aid to the understanding and controlling of complex processes. The widespread employment of pictorial instructions for e.g. assembling a piece of furniture, putting on a life jacket or tying a Windsor knot testifies to the extendibility of relative simple visual representations (Gombrich 1990).

The unifying element of such activities is space, the subject matter of architecture. It is therefore surprising that architectural design and analysis have invested little time and effort in explorations of the limitations in current representational approaches. Even in the face of practical impossibilities concerning the description of complex forms, the development of new and the refinement of existing representations has been assigned a low priority outside fundamental research. Practice and criticism seem more interested in continually reinventing roles and re-establishing the order of precedence for just a few existing techniques: sketching, orthographic projection, perspective projection and scale modelling.

The interests of practice also weigh on the issue of visual representation in academic research and teaching. One of the surprising consequences is the relative lack of rigorous representations for abstraction and analysis. It appears that architectural researchers are contented with diagrammatic simplification, as witnessed in typologic or programmatic analyses. Sometimes they even dispense with simplification, relying instead on the viewer's perceptual and cognitive powers for recognizing relevant information in a conventional architectural drawing.

The significance of visualization for architecture should also be viewed in the context of wider technocultural changes. Pictures are re-emerging as vehicles for the storage, manipulation and communication of information, especially in relation to the visual environment (Lopes 1996). Such changes are a useful antidote to the aesthetization of 
pictorial representation — of which design disciplines are often found guilty. 


\section{References}

Barrett, E., (ed) (1988). Text, ConText and HyperText: Writing with and for the computer. Cambridge, Massachusetts, MIT Press.

Barrett, E., (ed) (1989). The society of text. Cambridge, Massachusetts, MIT Press.

Biederman, I. (1987). "Recognition-by-components: A theory of human image understanding." Psychological Review 94(2): 115-147.

Biederman, I. (1995). Visual object recognition. Visual cognition. An invitation to cognitive science. 2nd ed. S. M. Kosslyn and D. N. Osherson (eds). Cambridge, Massachusetts.

Brachman, R. J. (1985). On the epistemological status of semantic networks.

Readings in knowledge representation. R. J. Brachman and H. J. Levesque (eds). Los Altos, Kaufmann.

Brachman, R. J. and H. J. Levesque (1985). Introduction. Readings in knowledge representation. R. J. Brachman and H. J. Levesque (eds). Los Altos, Kaufmann.

Eastman, C. M., Ed. (1975). Spatial synthesis in computer-aided building design. London, Applied Science.

Evans, G. and J. McDowell (ed) (1982). The varieties of reference. Oxford, Oxford University Press.

Evans, R. (1989). Architectural projection. Architecture and its image. E. Blau and E. Kaufman (eds). Montreal, Canadian Centre for Architecture.

Evans, R. (1995). The projective cast. Architecture and its three geometries. Cambridge, Massachusetts, MIT Press.

Gauchel, J., S. Van Wyk, et al. (1992). Building modeling based on concepts of autonomy. Artificial Intelligence in Design '92. J. S. Gero (ed). Dordrecht, Kluwer.

Gombrich, E. (1990). Pictorial instructions. Images and understanding. H. Barlow, C. Blakemore and M. Weston-Smith (eds). Cambridge, Cambridge University Press.

Goodman, N. (1976). Languages of art: An approach to a theory of symbols. Indianapolis, Hackett.

Gross, M. D. (1995). Indexing visual databases of designs with diagrams. Visual databases in architecture. Recent advances in design and decision making. A.

Koutamanis, H. Timmermans and I. Vermeulen (eds). Aldershot, Avebury.

Hodges, M. E. and R. M. Sasnett (1993). Multimedia computing. Case studies from MIT project Athena. Reading, Massachusetts, Addison-Wesley. 
Hoffman, D. D. and W. Richards (1985). "Parts of recognition." Cognition 18 65-96. Koffka, K. (1935). Principles of Gestalt psychology. New York, Harcourt Brace. Köhler, W. (1929). Gestalt psychology. New York, Liveright.

Koutamanis, A. (1995). Background information systems. Multimedia and architectural disciplines. B. Colajanni and G. Pelliteri. Palermo, ECAADE.

Koutamanis, A. (1995). Recognition and retrieval in visual architecural databases. Visual databases in architecture. Recent advances in design and decision making. A. Koutamanis, H. Timmermans and I. Vermeulen (eds). Aldershot, Avebury.

Koutamanis, A. (1996). Elements and coordinating devices in architecture: An initial formulation. 3rd Design and Decision Support Systems in Architecture and Urban Planning Conference. Part One: Architecture Proceedings. Eindhoven.

Koutamanis, A. (1997). Multilevel representation of architectural designs. Design and the net. R. Coyne, M. Ramscar, J. Lee and K. Zreik (eds). Paris, Europia Productions.

Koutamanis, A. and V. Mitossi (1992). Automated recognition of architectural drawings. Proceedings of the1 1th International Conference on Pattern Recognition. Los Alamitos, IEEE Computer Society Press. 1.

Koutamanis, A. and V. Mitossi (1993). "Computer vision in architectural design." Design Studies 14(1): 40-57.

Leeuwenberg, E. L. J. (1967). Structural information of visual patterns. An efficient coding system in perception. Doctoral dissertation, Catholic University of Nijmegen. The Hague, Mouton.

Leeuwenberg, E. L. J. (1971). "A perceptual coding language for visual and auditory patterns." American Journal of Psychology 84 307-350.

Lopes, D. (1996). Understanding pictures. Oxford, Clarendon.

Marr, D. (1982). Computer vision. San Fransisco, W.H. Freeman.

Mitchell, W. J. (1992). The reconfigured eye. Cambridge, Massachusetts, MIT Press.

Mitchell, W. J. and M. McCullough (1995). Digital design media. New York, Van Nostrand Reinhold.

Rosenfeld, A., (ed) (1984). Multiresolution image processing and analysis. Berlin, Springer.

Rosenfeld, A. (1990). Pyramid algorithms for efficient vision. Vision: coding and efficiency. C. Blakemore. Cambridge, Cambridge University Press. 
Shiffer, M. J. (1995). Multimedia representational aids in urban planning support systems. Understanding images. F. J. Marchese (ed). New York, Springer-Verlag.

Steadman, J. P. (1976). Graph-theoretic representation of architectural arrangement. The architecture of form. L. J. March. Cambridge, Cambridge University Press.

Steadman, J. P. (1983). Architectural morphology. London, Pion.

Stiny, G. and W. J. Mitchell (1978). "The Palladian grammar." Environment and Planning B 5 5-18.

Thompson, H. (1991). Tintin - Hergé and his creation. London, Hodder \& Stoughton.

Wertheimer, M. (1938). Laws of organization in perceptual forms. A source book of Gestalt psychology. W. D. Ellis (ed). London, Routledge \& Kegan Paul. 


\section{Visualization and architecture}

\section{The democratization of computer technologies}

The re-emergence of pictures as information carriers relates to the widely publicized assertion that we live in the computer era. Nevertheless, the high profile of computerization in most professions and in entertainment should not obscure that the computer is a very recent addition to daily life. A soberer assessment of current developments suggests that we are just entering an initial phase of the computer era. The most striking feature of this phase is the democratization of information technology. The middle and late nineties brought about dramatic changes in computerization. After two decades of relatively slow development which was moreover restricted to the initiated, the computer became a ubiquitous appliance linked to a new information infrastructure. Computerization of the workplace has been followed by an increasing presence of computers in entertainment and at home.

The wide availability of affordable computing power has been a significant factor for the application of information technology to the design and management of the built environment. As in every type of office, the proliferation of general purpose software for text processing, numerical calculation and database management and its promise of high efficiency has made the computer acceptable as a useful tool. The obvious next step has been the integration of domain specific systems, such as drafting, modelling and other visualization programs. This has proven more difficult and slower. The main reason has been the understandable caution with which we approach systems that purport to improve not only efficiency but also design quality and performance. Still, the professed practical advantages of such systems are such that the majority of architects and urban designers appears to be willing to espouse computerization.

While the computer's value in increasing efficiency has been amply proven, as for example in the production and management of building documents, its applications have yet to lead to higher quality and performance in designing the built environment or in the built environment itself. The availability of computational power is not matched by a methodical utilization of computing for the improvement of current practices. Most computing applications in architecture and planning are sporadic ad hoc transfers of technology which may resolve isolated problems but do little to relate the solutions they provide to their wider context. Such contrast with the general euphoria concerning the democratization of the computer also relates to the fact that the majority of designers working in practice with computers have not had the benefit of formal education in design computing. 


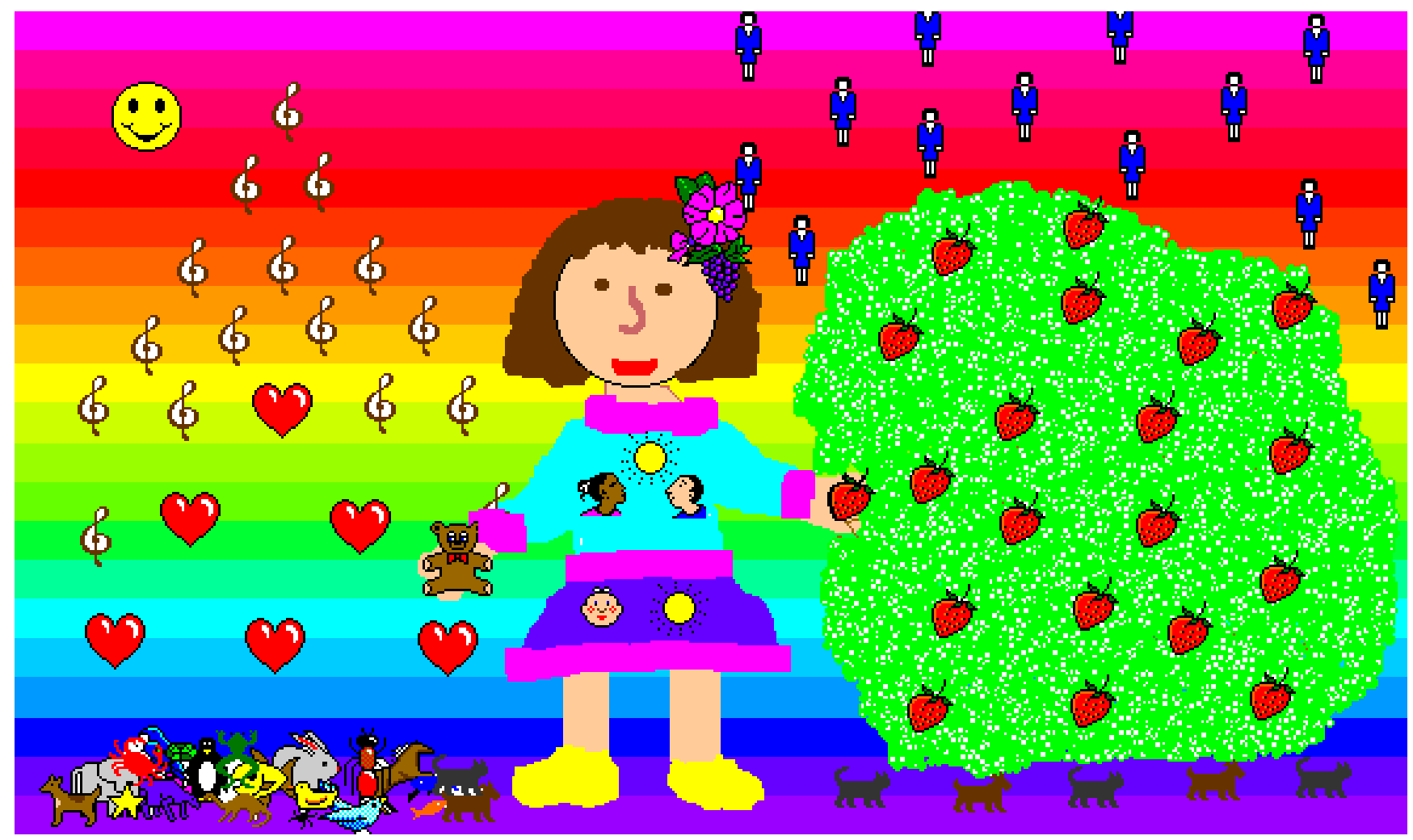

Figure 1. Drawing by a nine year old using KidPix on a Macintosh Powerbook 165c

Still, the new technologies are already having a profound influence on architectural visualization in two significant ways. The first is that, by making computational power available, affordable and relevant, they provide more efficient and economical implementations of pre-existing analogue techniques, as well as new, complementary tools. The younger generations are particularly proficient in digital visualization. Figure 1 is a casual drawing by a nine year old with the program KidPix on a Macintosh Powerbook 165c. Despite the added difficulty of having to master the trackball of the particular computer model, the drawing comes very close to the child's drawings on paper. Even the use of standardized elements in the computer program echoes her application of self-adhesive and stencilled figures.

The early acquaintance of today's children with computer visualization, their natural acceptance of computer cognitive and manual ergonomics, as well as their high exposure to related media, such as video, suggest that digital tools will soon cease to be an alien technology in architectural education and practice. Even thorny issues such as digital sketching (cf. figure 1) will be resolved simply by the future users' proficiency in both the digital and analogue versions. The new technology of automated recognition will further enhance the role of digital sketches by allowing seamless transition to two dimensional drawings and three dimensional models (Gross 1995; Koutamanis 1995). Similarly, possibilities of integration and communication offered by compound representations in hypermedia and multimedia will allow increased expressive power and coherence of visualization beyond the mere linking of different items and sorts of information.

The second influence of the democratization of computer technologies on architectural visualization lies in that it opens a wide and exciting new market for visualization in information systems. Current graphical interfaces are frequently developed in the direction of spatial forms, as for example the Internet with VRML. The architects' experience in representing spatial patterns visually has led to the assumption that the design of these interfaces and of interaction in information space adds to the scope of the architect who is arguably better suited to such subjects than other design specialists of today.

A more cautious evaluation of architectural knowledge and skills in visualization reveals a streak of 
arrogance. In the past the same arrogance has lead to unsuccessful excursions to industrial design, town planning, graphic design and practically every area that resembles architecture. The challenge of computerization presents a unique opportunity for refining and redefining the theory and practice of architectural visualization. We are currently lacking a unified theory of architectural representation that will dispense with the holistic, redundant and cumbersome principles that have been dominating modelling and information exchange. 


\section{Visualization and modelling}

\section{Representation}

A suitable working definition of what a representation is and what it does can be derived from (Marr 1982). According to this definition a representation is a formal system for making explicit certain entities in a transparent manner, i.e. together with an explanation of how the explicitness is achieved. The product of a representation as applied to a specific entity is a description. Familiar examples of representations include Roman and Arabic numerals (decimal or binary). Figure 2 contains alternative descriptions of the number 17 produced by different representations.

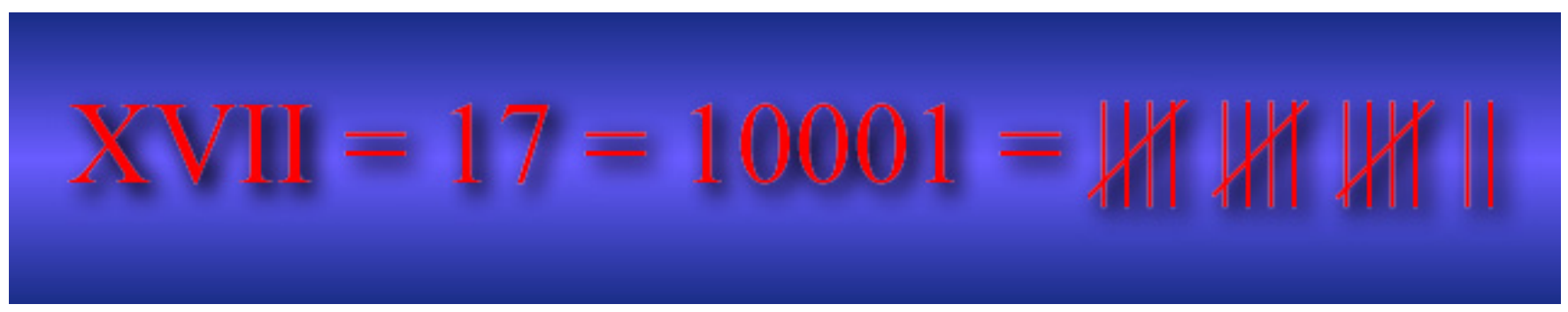

Figure 2. Alternative representations of a number

In each of these representations a number is described on the basis of a finite set of symbols and a rule systems for composing a description from the symbols. Arabic decimal numerals use the following set.

$$
\mathrm{S}_{\mathrm{A}}=\{0,1,2,3,4,5,6,7,8,9\}
$$

These symbols are correlated to a number in the following manner:

$$
\mathrm{n}_{\mathrm{n}} * 10^{\mathrm{n}}+\mathrm{n}_{\mathrm{n}-1} * 10^{\mathrm{n}-1}+\ldots+\mathrm{n}_{1} * 10^{1}+\mathrm{n}_{0} * 10^{0}=\mathrm{n}_{\mathrm{n}} \mathrm{n}_{\mathrm{n}-1} \cdots \mathrm{n}_{1} \mathrm{n}_{0}
$$

For example:

$$
1 * 10^{1}+7 * 10^{0}=17
$$

Arabic binary numerals make use of a smaller set of symbols and corresponding decomposition rules:

$$
\begin{gathered}
\mathrm{S}_{\mathrm{B}}=\{0,1\} \\
\mathrm{n}_{\mathrm{n}} * 2^{\mathrm{n}}+\mathrm{n}_{\mathrm{n}-1} * 2^{\mathrm{n}-1}+\ldots+\mathrm{n}_{1} * 2^{1}+\mathrm{n}_{0} * 2^{0}=\mathrm{n}_{\mathrm{n}} \mathrm{n}_{\mathrm{n}-1} \ldots \mathrm{n}_{1} \mathrm{n}_{0}
\end{gathered}
$$

For example:

$$
1 * 2^{4}+0 * 2^{3}+0 * 2^{2}+0 * 2^{1}+1 * 2^{0}=10001
$$

Architectural representations are essentially similar in structure. They consist of symbols for spaces and/or building elements, relations between the symbols and correspondence rules for mapping the symbols and their relationships to the subject of the representation. Figure 3 depicts the symbols of a basic set of building elements. The set is sufficient for describing orthogonal floor plans, such as the one in figure 4, as two dimensional arrays comprising generic building elements (Koutamanis 1995).

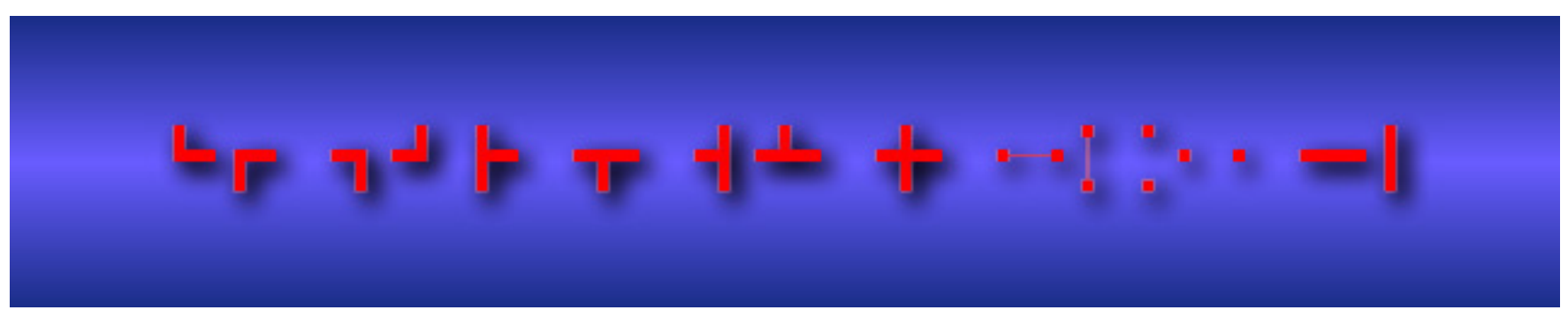

Figure 3. A basic set of symbols for floor plans 


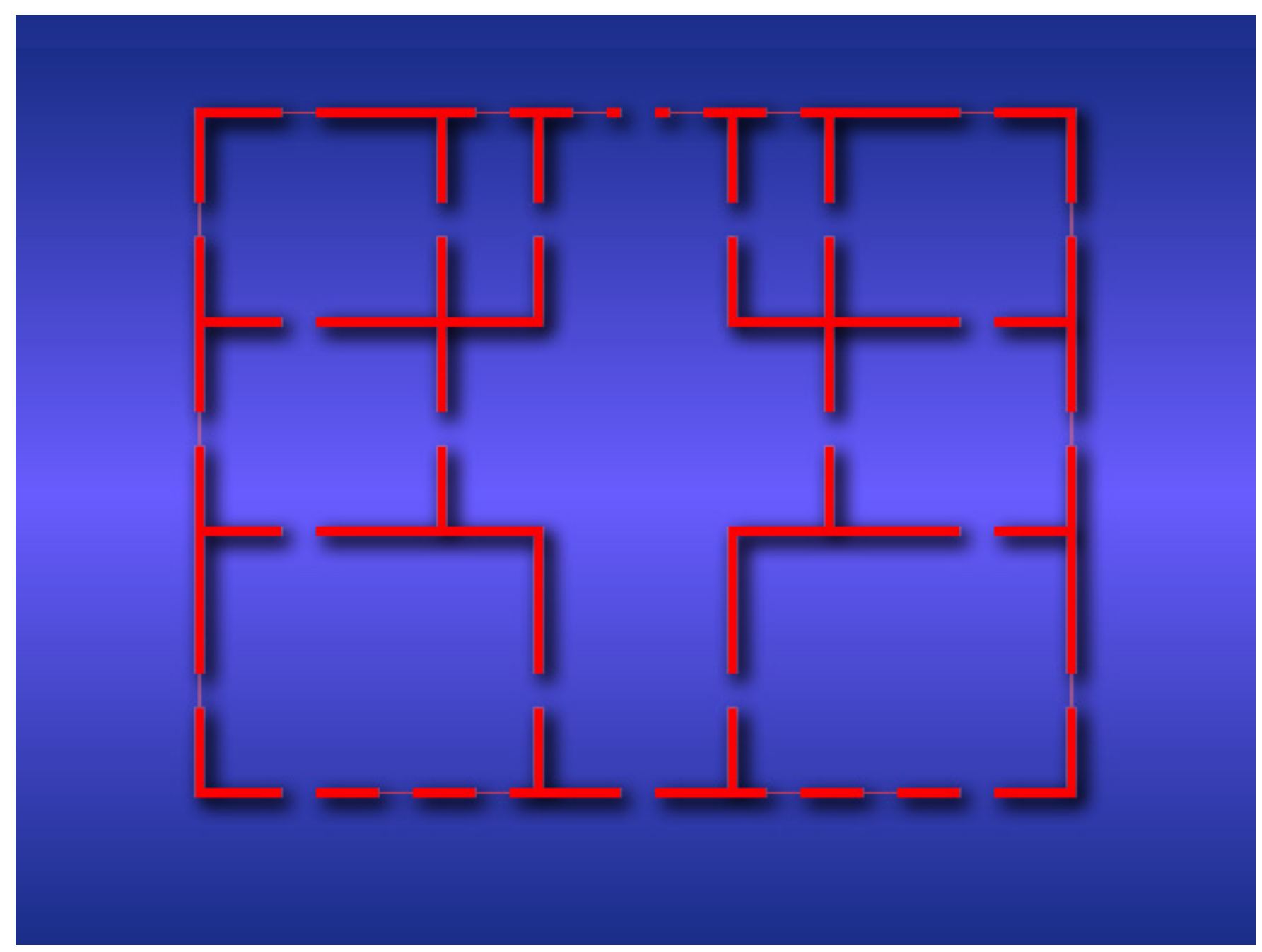

Figure 4. Floor plan created with the symbols of Figure 3

The choice of symbols relates to the constraints of the symbolized entities. These constraints are in turn reproduced in the representation. In the example of figures 3 and 4, there are specific expectations concerning the type of linked wall corners and junctions (the first nine symbols in figure 3). These expectations can be used to evaluate and control the syntactic correctness and integrity of a description. They can also be used to recognize the spaces in a floor plan (Koutamanis and Mitossi 1992; Koutamanis and Mitossi 1993; Koutamanis 1995).

Computational representations of architectural designs generally concentrate on these two types of symbols, building elements and spaces, i.e. the 'solids' and 'voids' of architectural composition. Most generative systems concentrate initially on the relative arrangement of spaces and subsequently on the positioning of building elements which bound the spaces (Eastman 1975; Stiny and Mitchell 1978). Other systems have focused on the

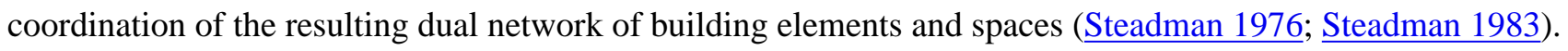




\section{Visualization and modelling}

\section{Implementation mechanisms}

The emphasis on spaces and building elements in academic research has not been matched in practical design computing and visualization. Drafting and modelling programs generally employ lower level geometric primitives, such as points, lines and simple surfaces, which signify the outlines of building components. Moreover, these geometric symbols are seldom grouped together into a coherent description of a component and have few if any explicit relations to other elements. Despite attempts to justify the choice of geometric primitives for architectural representations, it is obvious that they derive from analogue drawing practices.

A useful distinction, also derived from (Marr 1982), is between the representation and its implementation. For every representation there are several alternative implementations, usually depending on the context of the application. For example, binary numbers can be represented with Arabic numerals ( 1 or 0$)$ or with states of switches (ON or OFF). Both refer to the same representation, as it is the implementation mechanisms that change and not the actual symbols used in the representation.

The elevation of implementation mechanisms such as lines and surfaces to primitives of architectural design is symptomatic of two general conditions in the computerization of architecture. The first is that most digital techniques are direct transfers of analogue practices. This almost always includes unquestioning acceptance of the implementation mechanisms of an analogue representation as the basis of its digital equivalent. The second condition is an underlying mystification tendency which is confusing implementation mechanisms and visualization techniques with spatial form and its perception. The use of spaces and building elements as the basic primitives of architectural design representation is obviously too prosaic to allow for far-fetched associations and loose metaphors which can be easily accommodated in neutral geometric justifications.

The main consequence of the above is a weakening of the symbolic level of architectural representations and in particular of relationships between described entities or aspects. The absence of such relationships in digital architectural representations reduces the flexibility and adaptability of descriptions and makes interactive manipulation and partial automation unnecessarily complex and cumbersome. Techniques such as parametrization which rely on networks of relationships have yet to achieve popularity in practice merely because of the lack of explicit relevant entities and relationships between entities in digital design representations. 


\section{Visualization and modelling}

\section{Design modelling}

Traditionally the main application of visualization in architectural design has been the representation of built form, for the dual purpose of registering the input and output to cognitive processes (reinforcement of internal representations by external ones) and communicating design ideas (including the visual / geometric specification of forms to be built). The transition form analogue to digital media has been initially restricted to two dimensional representations (line drawings) which matched the limitations of available technology and the priorities of architectural practice. The subsequent addition of the third dimension to two dimensional drawings and the production of photorealistic renderings on the basis of three dimensional models was also geared to efficiency and productivity rather than new forms of expression (Mitchell 1992; Mitchell and McCullough 1995).

Despite significant insights into the logical structure of designs that have been produced by academic research, attachment to implementation mechanisms in digital representations has diminished the explicitness of symbolized objects, events and state of affairs. One possibility for the resolution of the problem lies in the tendency towards coordinated and interconnected representations in modelling, visualization and information systems. The practical advantages of following this tendency could promote a higher level of abstraction in the representation of built form, so as to correlate relevant, meaningful entities instead of some of the graphic elements used for depicting the entities.

Modern visualization technologies support attempts to unify architectural representations. The combination of the perceptual with the symbolic, the figurative with the abstract, the qualitative with the quantitative, takes two basic forms in computing. The first in the effortless, non-redundant transition from one representation to another and back. The direct communication between computer programs catering for representations as disparate as floor plans, photorealistic images and climatic analyses is a major preoccupation in current architectural computing. The second form is encountered in the compound representations which underlie multimedia and hypermedia systems. Despite their popularity, compound representations offer but a glimpse of their full potential with respect to the combination of different descriptions into a multidimensional system of sensory information. The symbiotic representational approaches in modern design computing are of significance for the future of conventional representations like floor plans and sections, whose resemblance to their subject is also product of the representational practice, i.e. becomes evident once one has learned the meaning of the representation (Goodman 1976; Lopes 1996). 
Another potential contribution of modern visualization technologies is the provision of sharper, more reliable and hopefully more intuitive geometric tools. The practical and conceptual necessity of describing three dimensional objects with coherence, accuracy and precision has created a strong but strained relationship between architecture and geometry. A frequent complaint is that orthographic projections may fail to register the salient features of their subject. The consequent rebellions against the "tyranny of the box" oscillate between giving up geometry altogether and adopting other, more complex geometries - choices whose outcome is never fully explored (Evans 1995).

Computer modelling is often criticized as being too restrictive with respect to the representation and creation of architectural form. The criticism (an interesting extension of similar remarks concerning orthographic and, to a lesser degree, projective projection) is certainly not unfounded but reflects more on human-computer interaction (including the user's understanding of geometric modelling with the computer) than on the modelling potential of the computer which are more than adequate for representing and presenting the few buildings in the history of architecture that have challenged analogue techniques. The addition of geometries not feasible without computers, such as fractals, improves further the expressive power of architectural visualization while asking for little more than comprehension of the underlying principles. 


\section{Visualization and modelling}

\section{Abstraction and specificity}

Research into the structure of symbolic representation has addressed on two main issues:

- which primitives should be employed and at what level (Brachman and Levesque 1985), and

- the possibility of units (chunks, partitions, clusters) more structured than simple nodes and links or predicates and propositions (Brachman 1985).

The primitives issue can be resolved by the analysis of existing representations of the built environment. These traditionally assume a direct, atomistic form. A conventional representation such as a map or a floor plan comprises atomic elements such as individual buildings or building components. These elements appear at an abstraction level appropriate to the scope of the representation. Depending upon the scale and purpose of a map, buildings are depicted individually or are catenated into city blocks. Similarly, a floor plan at the scale of 1:50 depicts building components and elements that are ignored or abstracted at 1:500. Most other aspects of built form remain implicit, with the exception of those indicated as annotations by means of colouring and textual or symbolic labels which convey information such as grouping per subsystem, material properties or accurate size. Relations between elements, such as the alignment of city blocks or the way two walls join in a corner are normally not indicated - unless of course they form the subject of the representation, as in detail drawings.

Academic research has extensively considered issues other than geometric information on shape, size and position of the components, notably the explicit representation of spatial primitives, spatial relationships and other entities relevant to the designer. Using formalisms such a semantic networks, frames, scripts and objects, academic research has produced associative symbolic which share the following features:

- a representation consists of objects and relations between objects;

- objects are described by their type, intrinsic properties and extrinsic relations to other objects;

- properties are described by constraints on parameters;

- relations are described by networks of constraints that link objects to each other.

Associative symbolic representations have been successful in the framework of highly focused generative systems where structure and intention can be controlled. More ambitious representations have attempted to integrate all relevant aspects and 
entities. Their main intention has been to resolve real design problems as encountered in practice. However, in most cases large or holistic representations have a size and exhibit a complexity that often render the representations unmanageable for both computers and humans. Problematic maintenance and lack of predictability in the behaviour of such representations, especially following modification and augmentation, severely limit their applicability (Gauchel, Van Wyk et al. 1992).

One approach to reducing complexity and improving flexibility in associative symbolic representations is based on the premise that spatial design representations are multilevel coordinated structures. Each representation level corresponds to a different abstraction level and possibly to different design aspects. While each level can be used as a self-sufficient representation, the coordination of all levels offers the flexibility and comprehensiveness required for tackling intricate and extensive problems. Coordination of levels can be based on the correspondence of elements, i.e. the existence and invariance of the same entities on multiple levels, as in the multiscale representations developed in computer vision (Marr 1982; Rosenfeld 1984; Rosenfeld 1990). Another form of coordination is based on the aggregation of constraints into coordinating devices that operate in conjunction with elements, as well as independently (as opposed to defining constraints on objects as fragmentary ad hoc relations). Such coordinating devices are either local and centred on elements or global and abstract (Koutamanis 1996; Koutamanis 1997). Examples of local coordinating devices are found in the constraint framework that underlies the positioning of architectural elements relative to each other. Global coordinating devices are often manifested as the grids, gratings and other schemata employed in typologic studies, comparative analyses and generative systems as abstractions of the overall spatial articulation of a design.

Integral to the multilevel representation approach are perceptual and cognitive mechanisms that determine our visual interaction with the built environment. These are involved at two related levels. The first concerns the identification of discrete three dimensional components of architectural form by means of the recognition-by-components theory (Biederman 1987; Biederman 1995). The second is the correlation of these components in the representation on the basis of the minimal coding principle of the structural information theory (Leeuwenberg 1967; Leeuwenberg 1971).

Once low level processing is completed, the first stage in the recognition of a scene is invariably a decomposition of its elements into simple parts, such as the head, the body, the legs and the tail of an a animal. The manner of the decomposition into parts does not depend on completeness and familiarity. An unfamiliar, a partly obscured animal or even a nonsensical shape are decomposed in a more or less the same way by all observers (Biederman 1987). The detection of where parts begin and end is based on the transversality principle which states that whenever two shapes are combined their join is almost always marked by matched concavities (Hoffman and 
Richards 1985). Consequently segmentation of a form into parts usually occurs at regions of matched concavities, i.e. discontinuities at minima of negative curvature. The results of the segmentation are normally convex or singly concave forms.

The recognition-by-components theory proposes that these forms actually constitute a small basic repertory of general applicability, characterized by invariance to viewpoint and high resistance to noise. The forms, called geons, are only 24 in number (Biederman 1987; Biederman 1995). The recognition-by-components theory appears to be as applicable to architectural scenes as to any other scene or object. Decomposition into geons is essentially similar to conventional decomposition into solid building elements and components. The main difference lies in the sensitivity of recognition-by-components to changes in the geometry within what is architecturally a single element. In most cases, however, an element that is decomposed into two or more geons is either a composite element, such as a wall with half columns or pilasters, or a geometrically complex object, such as a T- or L-shaped wall.

Gestalt psychologists have formulated a number of principles (or 'laws'), such as proximity, equality, closure and continuation, which determine the grouping of parts in a scene (Köhler 1929; Koffka 1935; Wertheimer 1938). Probably the most important and certainly the most mysterious of the Gestalt principles of perceptual organization is Prägnanz or figural goodness which refers to subjective feelings of simplicity, regularity, stability, balance, order, harmony and homogeneity that arise when a figure is perceived. Figural goodness ultimately determines the best possible organization of image parts under the prevailing conditions. As a result, it is normally equated to preference for the simplest structure. The principle is seen as the basis for preferring one our of several possible alternative descriptions of a percept.

The view of perception as information processing has led to attempts to formulate figural goodness more precisely. Given the capacity limitations of the perceptual system and the consequent necessity of minimization, it has been assumed that the less information a figure contains (i.e. the more redundant it is), the more efficiently it could be processed by the perceptual system and stored in memory. Arguably the best model in this line of investigation has been the structural information theory (Leeuwenberg 1967; Leeuwenberg 1971). According to it, a pattern is described in terms of an alphabet of atomic primitive types, such as straight line segments and angles at which the segments meet. This description (the primitive code) carries an amount of structural information that is equal to the number of elements (i.e., instances of the primitives) it contains. The structural information of the primitive code is subsequently minimized by repeatedly and progressively transforming the primitive code on the basis of a limited number of coding operations: iteration, reversal, distribution and continuation. The coding process returns the end code, a code whose structural information cannot be further reduced. The structural information of a pattern is that of its end code.

Recognition-by-components and structural information theory provide the basis for: 
- recognizing and representing the solid elements of an architectural scene;

- grouping the recognized elements in multiple alternative configurations;

- evaluating the alternative configurations with respect to coding efficiency; and

- establishing preference for one or two dominant configurations which represent the intuitively acceptable or plausible interpretations of the scene.

These operations link the representation of the built environment with perception and figural goodness. The necessary deviations from established conventional architectural representations reflect the choice of general cognitive and perceptual theories as the starting point of the investigation. Architectural representations and in particular (a) the use of outlines to denote solid entities and spaces and (b) the deterministic decomposition into known components should be reconsidered with respect to the recognition-by-components theory and related vision research. The grouping of these components into a coherent description should rely on structural information theory, especially with respect to establishing preference for one group configuration over its alternatives. 


\section{Visualization and modelling}

\section{Compound representations}

Probably the most interesting new challenge in computerization is presented by multimedia and hypermedia. Starting with such specific problems as the role of computing in technical documentation and networked online systems for collaborative work and information retrieval (Barrett 1988; Barrett 1989), they have developed through the Internet into a ubiquitous infrastructure (Hodges and Sasnett 1993; Koutamanis 1995). This infrastructure has supplemented tools for the design and analysis of the built environment by integrating their output as well as contextual information necessary for interpreting and utilizing this output (Shiffer 1995). The compound representation formed by the integration constitute a new paradigm that is applicable at practically all levels of specificity. The combination of all available and relevant descriptions creates immersive, multifaceted and utlimately self-contained representational systems which arguably offer the most fruitful environment for the implementation and use of modular coordinated design representations. 


\section{Realism: visual inspection and analysis}

\section{Projecting appearances}

The difference between pictorial and other descriptions (e.g. textual) is commonly explained on the basis of resemblance. A picture represents a subject by the intended resemblance of its pictorial properties to the visual perception of its subject. Some interpretations of resemblance may lead to limited views, such as the assimilation of the experience of seeing a picture to the real life experience of seeing the picture's subject, which moreover are unrelated to the symbolic structure of a picture's content (Goodman 1976; Evans and McDowell 1982; Lopes 1996). Nevertheless, resemblance remains an appropriate vehicle for investigating perceptual and cognitive issues involved in visual representation.

Architectural visualization has been rather ambivalent with respect to the resemblance issue. On the one hand, most basic design representations combine orthographic projection of canonical views with conventional symbolization. On the other, axonometrics, isometrics, perspectives and especially the rendered ones consciously attempt to project or reconstruct a veridical visual experience. This ambivalence stresses the correspondence of composition and projection in architecture to Euclidean and projective geometry. In both architecture and geometry a historical shift can be detected from measurement and accurate representation of a picture's subject to the picture itself (Evans 1995).

The proliferation of affordable computer tools for photorealistic visualization has been placing even more emphasis on the architectural picture. The connectivity of these tools to the standard CAD documentation of design practice means that computer-rendered photorealistic perspectives are often used instead of simpler images which would convey the same information, especially when the photorealistic version includes too many assumptions concerning colour and material. It is perhaps ironic that some of the more interesting additions to computer visualization include references to simpler rendering techniques from the past. For example, figure 6 has been rendered with the Illustrator 2 plug-in for 3D Studio MAX. In their attempt to reproduce the quality of colouring and backgrounds in comic books, e.g. the ones Edgar P. Jacobs created for Hergé's Tintin albums (Thompson 1991), such techniques are an alternative to the standard, almost-photorealistic renderings (figure 5). The abstraction of comic book imagery is arguably better suited to most stages of the design process, as well as to human recognition of built form. 


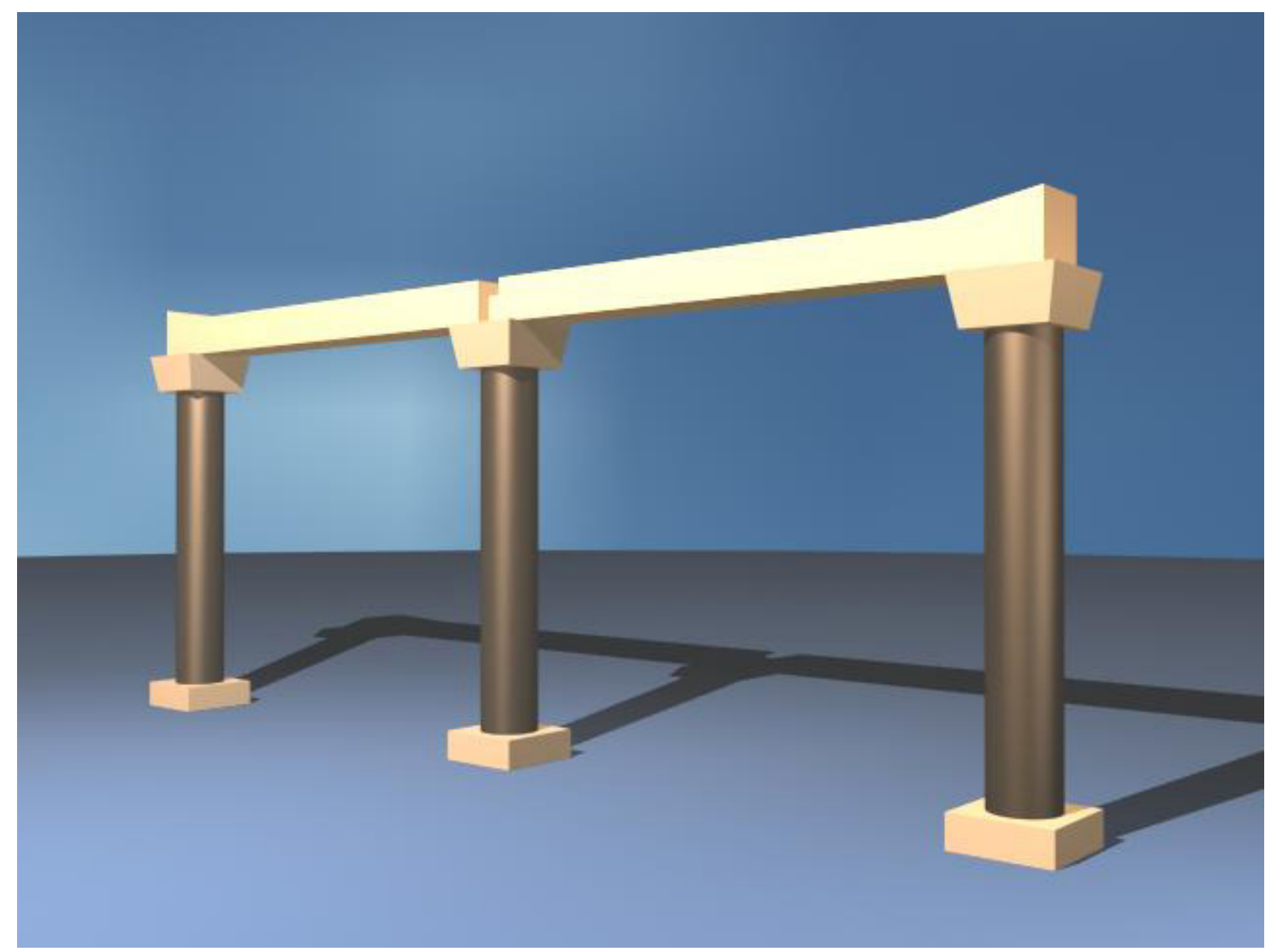

Figure 5. Image produced with the standard 3D Studio MAX renderer 


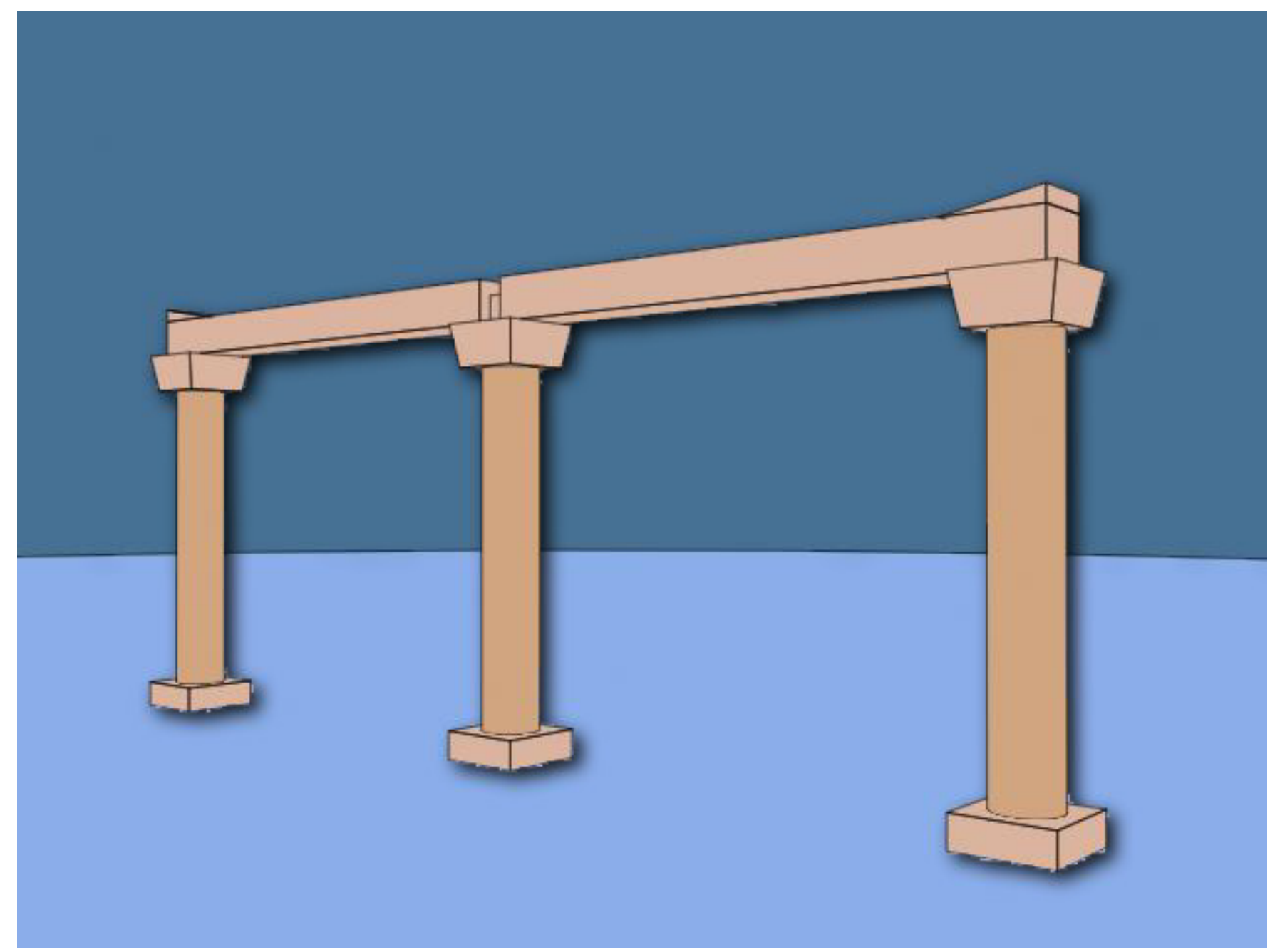

Figure 6. Image rendered with the Illustrator 2 plug-in for 3D Studio MAX 


\section{Realism: visual inspection and analysis}

\section{Beyond intuition: scientific visualization}

Design analysis has been traditionally performed with normative rule-based systems geared to generative approaches. The numerous dissections of the design process have resulted into a multiplicity of models which attempt to describe the steps a designer takes in her quest for a satisfactory solution. Most models also aspire to prescribe the optimal sequence of design actions. What they propagate is a form of orthopraxy (as opposed to the orthodoxy of formal systems such as the Classicism and the Modernism). Their underlying assumption is that if one follows the sequence of design stages prescribed in the model, she can arrive at a design that satisfices the programmatic requirements.

It is unfortunate that no such model to-date can match the intuitive performance and creativity of the human designer. Being based on metaphors and similes, most models do little beyond explain a few specific aspects of designing. Moreover, while they may improve the designer's awareness of actions and decisions, they seldom lead to the development of new, sharper tools for higher effectiveness and reliability in the face of today's complex design problems. Perhaps the main reason for the scarcity of such tools lies in the relative lack of interest in the analysis of design products.

Historically such analysis has been subservient to synthesis. Long before terms such as functional and programmatic analysis were invented, buildings and design decisions were being parsed towards an identification of their causes and effects. These were subsequently formalized into rules and stereotypical "good" solutions which served as the basis of most building regulations and design textbooks. Rules and stereotypes have mostly a proscriptive function. They attempt to offer design guidance by pointing out errors and inadequacies, i.e. what falls short of the established norms.

The proscriptive approach also underlies computational studies which focus on the analysis of designs using the same or similar rules transformed into expert or knowledge-based systems. In these a design is described in a piecemeal fashion which permits correlation of the relevant aspects or factors with the rules. The end product of the analysis is an acceptability test based on the matching to the constraints of the solution space. The added value of such systems lies in the provision of feedback which facilitates identification of possible failure causes.

Design analysis is moving towards a new paradigm, based more on simulation than on abstractions derived from legal or professional rules and norms. Recent developments in areas such as scientific visualization provide advanced computational tools for achieving high detail and exactness, as well as feedback for design guidance. The close correlation of photorealistic and analytical representations (figures 7 and 8) clarifies and demystifies the designer's insights and intuitions. Moreover, the combination of intuitive and quantitative evaluation offers a platform of effective and reliable communication with other engineers who contribute to the design of specific aspects, as well as comprehensible presentation of projected building behaviour and performance. 


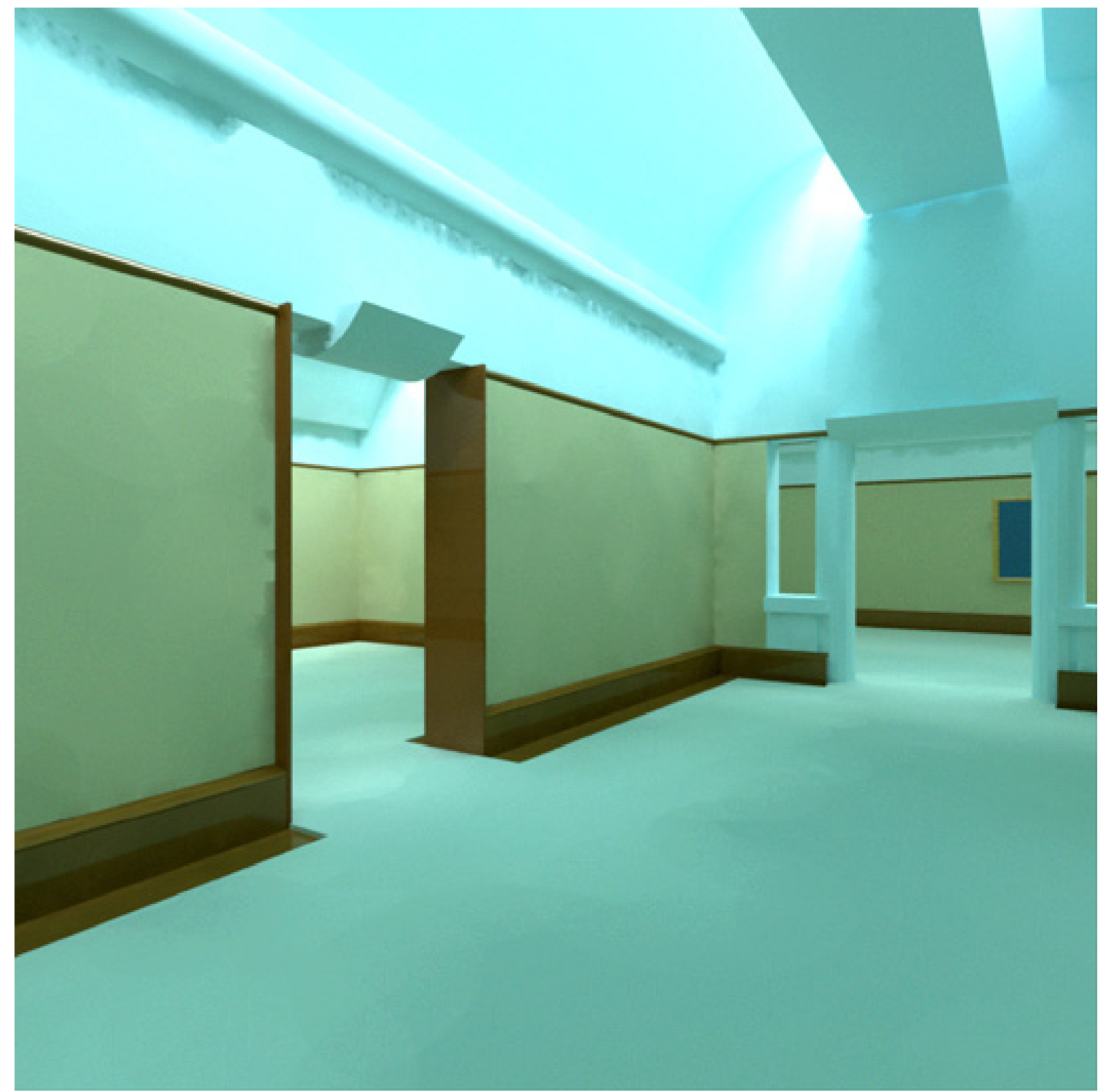

Figure 7. Photorealistic light simulation (Radiance image by A.M.J. Post) 


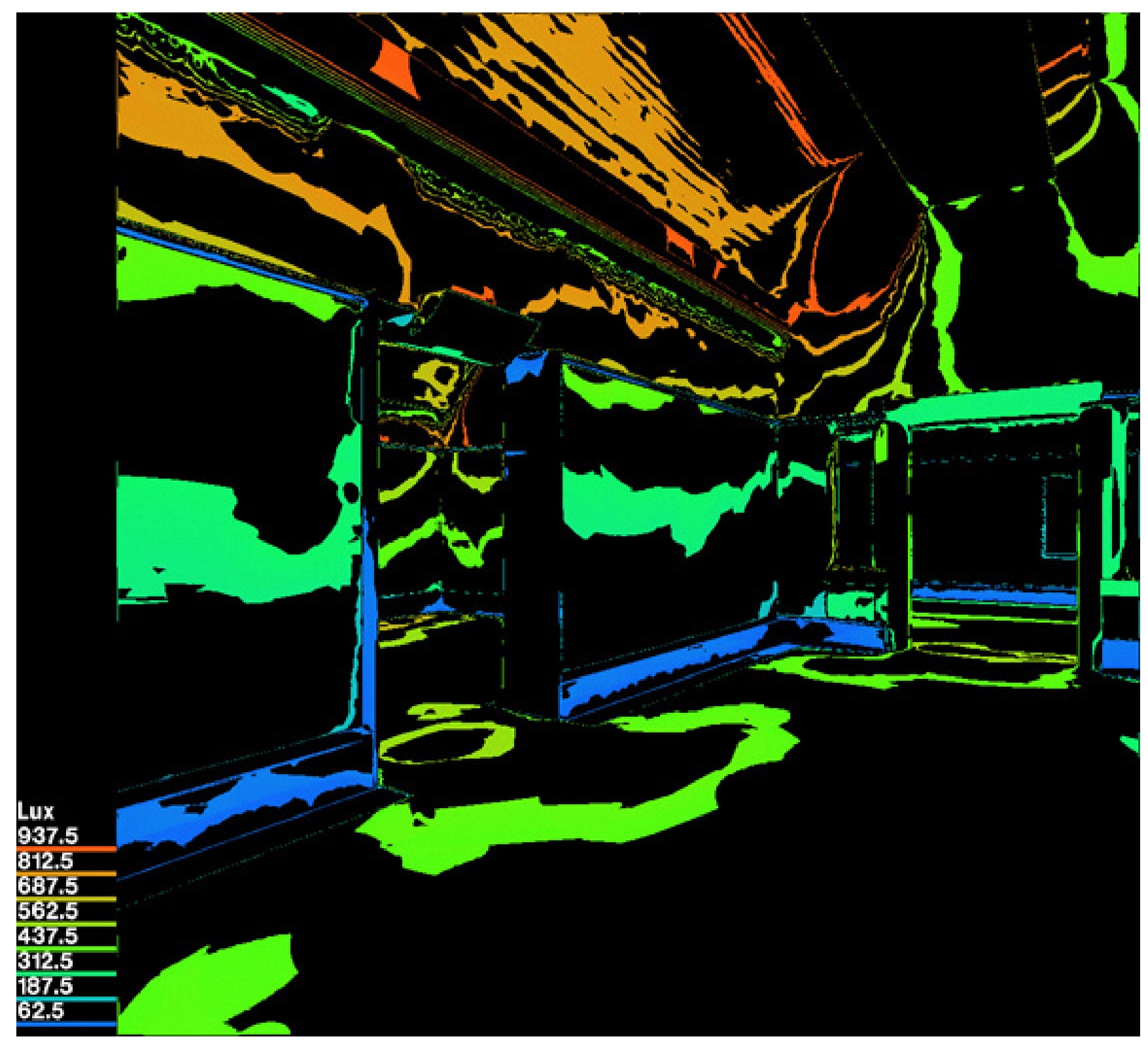

Figure 8. Light simulation: intensity analysis in the space of figure 1 (by A.M.J. Post) 


\section{Realism: visual inspection and analysis}

\section{Dynamic visualization}

Dynamic visualization is often presented as the pinnacle of architectural representation, the fullest form of visual realism. By including movement of one sort or another in a three dimensional representation the designer adds depth and time to the subject under controlled conditions, i.e. in the framework of a specific event or state. As a dynamic description is a sequence of static, normally photorealistic images the results can be superior to other representations for visual inspection, analysis and communication.

As with photorealism, a frequent argument for dynamic visualization is the ease by which it can be produced out of three dimensional design representations. While this is true for simple, undemanding movements of the camera or in the scene, more complex subjects and presentation techniques require knowledge and skills beyond the scope of architecture. These are best found in filming and range from camera positioning and movement to lighting and to editing, mixing and visual effects. The technical aspects are largely integrated in the digital tools, but the architect must effectively step into the film director's chair so as to coordinate, guide and manage the process.

Directing a dynamic description is a role that a role that in principle befits the architect as the specifier and coordinator of design and construction of a project who does not necessarily have any physical involvement in the actual building. However, the fulfilment of the role necessitates substantial transfer of filming knowledge that complements the technical possibilities of digital dynamic visualization. Ironically most of this knowledge refers to techniques for reproducing on film environments and events without actually having the camera there and then. Even when shooting on location artificial lighting and sets are used to enhance resemblance to the scene envisaged in the script. In the studio everything is not only artificial but also opportunistically fragmented so as to minimize cost without loss of effectiveness and efficiency. The techniques involved in making a coherent and believable seuqence of images from short takes of such fragments and illusions forms the core of the knowledge that has to be integrated in architectural visualization. Several techniques have already been adopted in architectural design. Matting, for example, is nowadays widely used in making composite images from rendered perspectives of new designs and photographs of their prospective sites.

The main problem with filming techniques is that they run contrary to the holistic undercurrent of architectural design and CAAD. The use of partial models for different aspects and abstraction levels does not agree with the idea of a single, complete and integral three dimensional representation for the whole design. On the other hand, a multilevel modular representation is capable of accommodating the practicalities of dynamic architectural visualization without sacrificing the coherence and consistency of the representation.

Most filming techniques are born out of necessity. However, they are not restricted to compensating for practical limitations. They also offer the means for constraining and controlling a process. One such device is the story board, a series of annotated drawings, essentially similar to a comic strip (figure 9). The drawings depict the découpage of the film, i.e. its structure in terms of takes and camera positions and movements. The application of storyboarding in architectural visualization on the basis of a modular coordinated representation adds a vertical coordinating device responsible for specific aspects arranged in a sequential manner. 

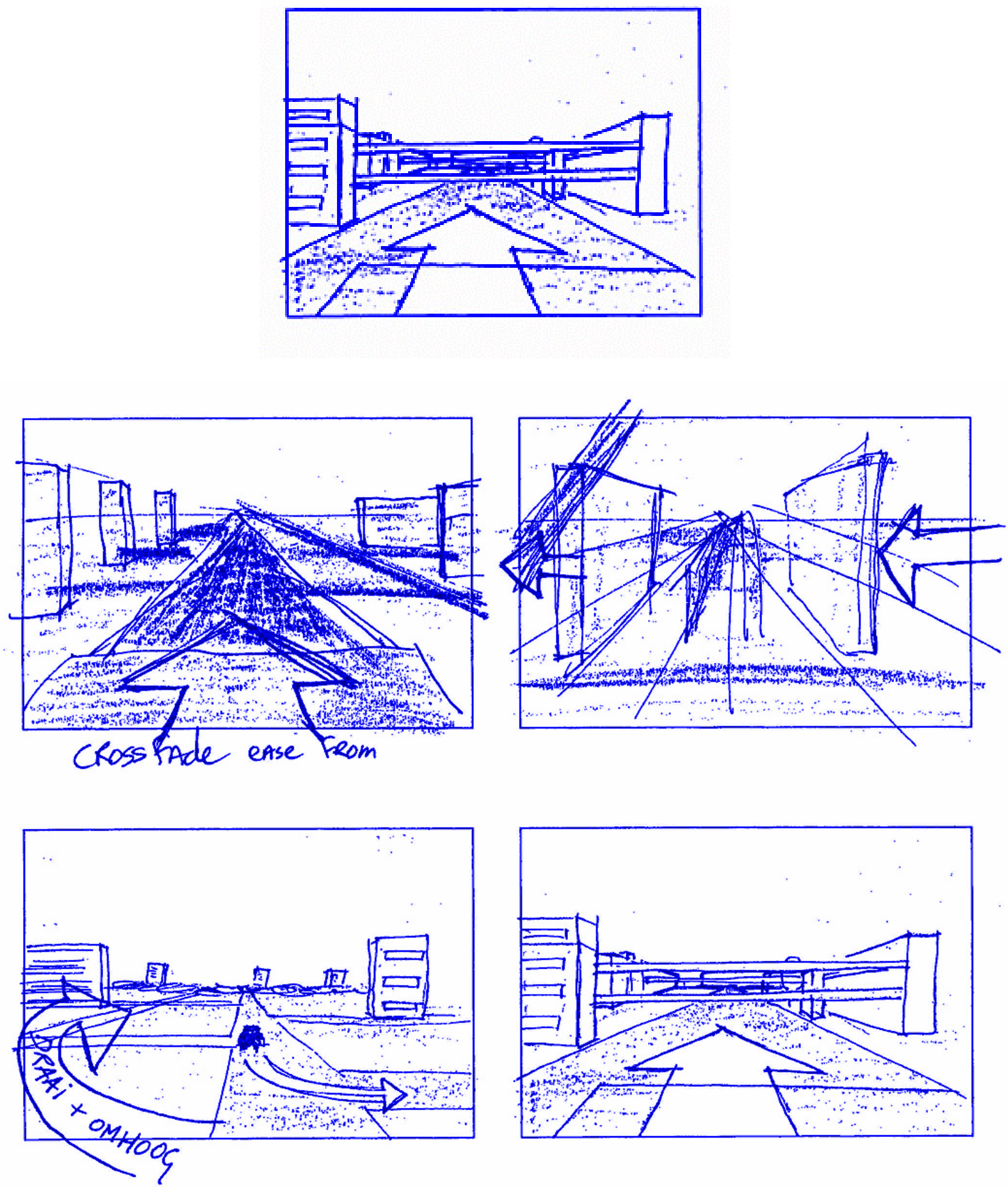

Figure 9. Story board extract (by I.R. van 't Hof) 


\section{Future developments}

One of the toughest exercises in design computing is predicting future developments. The race for more computational power, the availability of increasingly more worthwhile computer programs and improvements in operating systems are just a few of the factors which make the projection of current technical aspects rather futile. To a lesser degree this also applies to approaches and methods, especially those aspects that are inescapably constrained by technical opportunity, such as human-computer interaction. All that remains is fundamental issues which should guide technological development.

Probably the most significant fundamental issue for architectural visualization is representation. Current visualization problems are posing a wide spectrum of questions concerning our representational assumptions. Rather than adapting design representations to the requirements of these problems, we should attempt to arrive at a unifying theory of representation capable of dealing with such problems in a systematic, consistent manner. The main objective of this theory should be other than the current goal of increasingly higher levels of photorealism: the inclusion of modelling and analytical facilities which support and enhance the architect's intuitive creativity without prescriptive or proscriptive restrictions. 